\title{
Improving cancer imaging with magnetic nanoparticles: where are we now?
}

\author{
"In the past years, the imaging aspect of \\ magnetic nanoparticles has been discussed \\ several times."
}

First draft submitted: 25 October 2016; Accepted for publication: 8 November 2016; Published online: 12 January 2017

Keywords: cancer $\bullet$ imaging $\bullet$ magnetic nanoparticles $\bullet$ MPI $\bullet$ MRI

As cancer remains still one of the major causes of death, its precise diagnosis is of invaluable importance. Imaging is recognized as pivotal to the management of patients with cancer. However, these requirements meet groundbreaking developments not only in MRI and radiology but also in many other fields in imaging techniques. Nanotechnology, which has taken an impressive development in the past decades even in partial aspects like magnetic nanoparticles, could contribute essentially to this issue. Big expectations and great chances lead to the question: what is the score in cancer imaging with magnetic nanoparticles: where are we now in 2016?

\section{Requirements for suitable cancer imaging}

None of the already available clinical imaging modalities covers the entire range of requirements. In the past years, the imaging aspect of magnetic nanoparticles has been discussed several times. The advantages and limitations of each imaging technique have been described extensively [1]. A major question is to which question fits which imaging method. Husband and Rezneck have noted principles for choosing the right imaging technique, that seems to be still a helpful orientation [2]. Thus, each methodology has to be evaluated due to certain aspects that are briefly enumerated. The 'technical performance' is the ability to obtain high-quality signals in a reasonable time frame. 'Diagnostic performance' is stated as identifying the disease correctly. It is concerned to consist of sensitivity, specificity, positive predicted value and accuracy in a certain clinical situation. The matter of 'diagnostic impact' arises by the influence of the result of the applied method in the context of the clinician's diagnostic confidence. In other words, does the physician trust the images due to his one experience and in addition to other disease pattern. The 'therapeutic impact' reflects alterations in the treatment of patients due to the results of imaging. The last point to be mentioned, 'impact on health,' is very complex for evaluation since it faces aspects like public health cost, synergistic or adverse effects with other disciplines, which cannot be captured by the applier so easily.

Regarding the multitude of imaging techniques we like to focus on those applications which could obviously have a beneficial effect if they are combined with magnetic nanoparticles. Imageable magnetic nanoparticles could also carry payloads of active ingredients to fight tumor tissue and therefore they can be considered as 'theranostics'. Unfortunately, this aspect is too extensive to be discussed at this place. It is useful for the consideration to classify whether intrinsic properties of magnetic nanoparticles cause the imaging signals or whether another imaging agent for this purpose is required.

\section{Magnetic nanoparticle imaging utilizing their intrinsic properties} Intrinsic properties to be utilized are due to the magnetic properties of iron oxide which

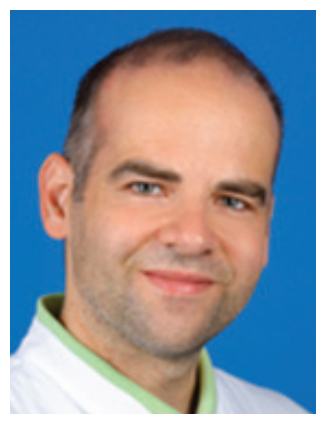

Rainer Tietze

ENT Department, Section for Experimental Oncology \& Nanomedicine (SEON), Else Kröner-Fresenius-Stiftung Professorship, University Hospital Erlangen, Erlangen, Germany

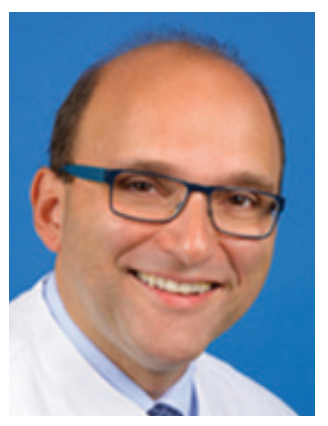

Christoph Alexiou

Author for correspondence: ENT Department, Section for Experimental Oncology \& Nanomedicine (SEON), Else Kröner-Fresenius-Stiftung Professorship, University Hospital Erlangen, Erlangen, Germany c.alexiou@web.de 
can be deployed for MRI. From unwanted artifacts, derived from cosmetics [3], to a first generation of super paramagnetic iron oxide nanoparticle (SPION)-based MRI contrast agents lasts the development history and those substances were primarily intended for imaging of tissues of the rethiculoendothelial system [4]. The commercialization begun and a couple of formulations were available in the late 1990s of the last century [5]. In principle, affecting both relaxation modalities $\mathrm{T} 1$ and $\mathrm{T} 2$, the main aspect was always to enhance the T2 or respectively $\mathrm{T} 2 *$ relaxation. Although the physical backgrounds of $\mathrm{T} 1$ relaxivity for small SPIONs were already understood, they did not play a crucial role for the development of particle-based MRI contrast agents. In most cases, it is the SPION's magnetic susceptibility and therefore the capacity to reduce the magnetic resonance signal which was utilized together with special spin-echo sequences to generate 'dark' image signals.

Only a few years later, most of those first-generation SPION-based magnetic resonance agents were already withdrawn from the market or they did not reach further development stages during clinical approval procedures. Some say, limited application fields and deficient diagnostic impact together with economic considerations were the main reasons why the formulations were deduced from the markets. In principle, they failed in competing with contrast agents which base on Gd-complexes. But, objective statements are scarce and a comprehensive discussion on the failure of the first-generation SPION-MRI agents would go far beyond the scope of this editorial.

Interestingly, this failure goes along with a new rise in attraction of SPIONs for MRI [6]. New perspectives like T1-weighted imaging are also discussed as cell tracking in the context of macrophages infiltration into tumors $[7,8]$.

The convincing advantage of SPIONs as MRI contrast agents in comparison with Gd-based substances lies in their easier use for functional imaging. A series of promising applications arose in the recent years. Some examples shall be given at this point: Shevtso et al. developed SPIONs which are decorated with a recombinant IL-1 receptor in order to target glioma cells in vivo [9]. Breast cancer is the target of another SPIONs formulation which are functionalized with proline-rich peptides which can bind preferably to estrogen-receptor-positive cancer cells [10]. Moreover, folic acid functionalization of SPIONs is a well-known strategy to address tumor cells which overexpress the respective folic acid receptor. This is utilized for SPION-based MRI by Sanjai et al. [11]. A couple of further applications are highlighted by Bakhtiary et al. with a focus on early detection of cancer using SPIONs and MRI [12].
Another argument for the (re)entry of SPIONs for MRI is the fact that Gd-based contrast agents are under critical discussion due to possible adverse side effects [13].

A relatively new technique, called magnetic particle imaging (MPI), attracts increasing attention as it detects SPIONs in an extraordinary sensitive manner and with short acquisition times which allows dynamic imaging applications. An extensive overview of current perspectives and a clear explanation about the technique itself is given by Pablico-Lansigan et al. [14]. Many aspects concerning tumor-specific imaging of MRI using functionalized SPIONs are also applied to MPI. Beyond that, tracking of metastasis and control of surgical treatments in oncology are even more promising applications for MPI. Another example of utilizing intrinsic properties of magnetic nanoparticles for imaging is magnetorelaxometry which uses the Néelrelaxation signal [15]. The method is extraordinarily sensitive but lacks precise spatial resolution. Main disadvantage of both MPI and magnetorelaxometry is that anatomic information cannot be depicted. Therefore, future clinical application must include MRI.

\section{Magnetic nanoparticle based imaging by additional imaging agents}

SPION-based imaging, whereby the imaging signal comes from additional agents like radio tracers or fluorescence marker, is a manifold issue. Applications, expectations and outcomes vary in a wide range and a general comment on that is nearly impossible. Nevertheless, a few words about this issue shall be written. Of course, there are plenty ways to make SPIONs visible. But their application is neither easy nor their respective formulation is suitable for general biomedical purposes. Radiolabeling for positron emission tomography or single-photon emission computed tomography imaging requires high regulatory, and technical efforts and labeling strategies have to be fast and reliable to avoid low radio chemical yields and insufficient specific activities. Optical imaging and therein fluorescence imaging, which is by far the most investigated optical imaging technique is, in principle, easier to perform than radiolabeling in terms of handling, technical infrastructure and regulatory issues. But, there are of course some considerable limitations. Most important is the limited range of light emission after excitation in tissues. Another disadvantage is the difficulty of quantification of probe accumulation which compromises obligate information about the state of tumor progression. This obstacle requires more sophisticated settings like fluorescence diffuse tomography, for instance [16]. This imaging technique, of course, does not display any anatomical 
information and requires additional imaging modalities to allocate the signals in the respective anatomic region.

\section{Conclusion}

Imaging of tumors can be both viewed from the standpoint of basic research and from the perspective of more application-oriented research. Here, of course, apply different standards. Methods for magnetic nanoparticle based imaging of tumors which are already clinically approved or not far away from their clinical implementation should be able to rely on already established technical equipment. This is the reason why for the new approaches of SPIONs for MRI, a surpassing importance is attached. It is the only method which works on its own and needs no further imaging method in principle. The suitability to detect primary tumors and metastasis reliably has been already proven [17]. Of course, MPI, radiolabeling and fluorescence labeling as mentioned above and furthermore currently investigated techniques like ultrasound [18] and various optic acoustic imaging techniques [19] could leverage the diagnostic outcome of an MRI setting which uses magnetic nanoparticles as imaging agents. These additional techniques would make especially sense if the diagnostic performance and the related diagnostic impact of MRIonly imaging is inadequate and if a hybrid imaging, combining MRI and positron emission tomography, for instance, system shows much beneficial diagnostic outcome [20]. But developers of those techniques have to answer the question whether they aim for clinical applications or remain on a status which is mainly classified by gaining scientific knowledge. It is not only the imaging apparatuses which have to be shifted to the status of medicinal products but also the nanoparticles as contrast agents which have to be produced under current GMP conditions which are necessary for clinical trials.

\section{References}

1 Condeelis J, Weissleder R. In vivo Imaging in Cancer. Cold Spring Harb. Perspect. Biol. 2(12), a003848 (2010).

2 Husband J, Reznek R. Imaging in Oncology. Taylor \& Francis Group, FL, USA. 1 (2004).

3 Lund G, Wirtschafter JD, Nelson JD, Williams PA. Tattooing of eyelids: magnetic resonance imaging artifacts. Ophthalmic Surg. 17(9), 550-553 (1986).

4 Gandon Y, Brunet F, Guyader D, Brissot P, Carsin M, Simon J. Super-paramagnetic iron oxide: an MRI contrast media for the reticuloendothelial system. Ann. Radiol. (Paris) 32(4), 267-272 (1989).
Concerning magnetic nanoparticles for MRI, the T1, T2 and $\mathrm{T}^{*}$ relaxivities are as important as the reliability and reproducibility of respective synthesis of nanoparticles. Certain quality standards have to be guaranteed which make it possible to transfer the substances into clinical trials. Of course, this applies for any drug, but sophisticated surface-functionalized nanoparticles for not-established imaging techniques have more chances to fail during preclinical development than magnetic nanoparticles for MRI.

\section{Outlook}

The issue of cancer imaging with magnetic nanoparticles is prospering but extensive and complex. As scientists in the field of biomedical development and application of nanotechnology with a strong link into the clinical practice, we like to summarize the topic the following way: magnetic nanoparticles have intrinsic properties which can be utilized for MRI and MPI. Imaging agents on the surface of particles enable further imaging modalities like fluorescence or radiolabeling. From the viewpoint of closeness of clinical application, currently, MRI is superior to any other magnetic nanoparticle based imaging modality.

\section{Financial \& competing interests disclosure}

The authors like to thank the EU 'NanoAthero' project FP7NMP-2012-LARGE-6-309820 for funding. The authors have no other relevant affiliations or financial involvement with any organization or entity with a financial interest in or financial conflict with the subject matter or materials discussed in the manuscript apart from those disclosed.

No writing assistance was utilized in the production of this manuscript.

\section{Open access}

This work is licensed under the Attribution-NonCommercialNo Derivatives 4.0 Unported License. To view a copy of this license, visit http://creativecommons.org/licenses/by-nc-nd/4.0/

5 Wang YX, Hussain SM, Krestin GP. Superparamagnetic iron oxide contrast agents: physicochemical characteristics and applications in MR imaging. Eur. Radiol. 11(11), 2319-2331 (2001).

6 Jin R, Lin B, Li D, Ai H. Superparamagnetic iron oxide nanoparticles for MR imaging and therapy: design considerations and clinical applications. Curr. Opin. Pharmacol. 18, 18-27 (2014).

7 Senpan A, Caruthers SD, Rhee I et al. Conquering the dark side: colloidal iron oxide nanoparticles. ACS Nano 3(12), 3917-3926 (2009).

8 Weissleder R, Nahrendorf M, Pittet MJ. Imaging macrophages with nanoparticles. Nat. Mater. 13(2), 125-138 (2014). 
9 Shevtsov MA, Nikolaev BP, Yakovleva LY et al.

Recombinant interleukin-1 receptor antagonist conjugated to superparamagnetIc iron oxide nanoparticles for theranostic targeting of experimental glioblastoma. Neoplasia 17(1), 32-42 (2015).

10 Ozdemir A, Ekiz MS, Dilli A, Guler MO, Tekinay AB. Amphiphilic peptide coated superparamagnetic iron oxide nanoparticles for in vivo MR tumor imaging. RSC Adv. 6(51), 45135-45146 (2016).

11 Sanjai C, Kothan S, Gonil P, Saesoo S, Sajomsang W. Super-paramagnetic loaded nanoparticles based on biological macromolecules for in vivo targeted MR imaging. Int. J. Biol. Macromol. 86, 233-241 (2016).

12 Bakhtiary Z, Saei AA, Hajipour MJ, Raoufi M, Vermesh $\mathrm{O}$, Mahmoudi M. Targeted superparamagnetic iron oxide nanoparticles for early detection of cancer: possibilities and challenges. Nanomed. Nanotechnol. 12(2), 287-307 (2016).

13 Forghani R. Adverse effects of gadolinium-based contrast agents: changes in practice patterns. Top. Magn. Reson. Imaging 25(4), 163-169 (2016).

14 Pablico-Lansigan MH, Situ SF, Samia ACS. Magnetic particle imaging: advancements and perspectives for realtime in vivo monitoring and image-guided therapy. Nanoscale 5(10), 4040-4055 (2013).
15 Schwarz M, Dorfler A, Engelhorn T et al. Imaging modalities using magnetic nanoparticles - overview of the developments in recent years. Nanotechnol. Rev. 2(4), 381-394 (2013).

16 Kleshnin M, Shirmanova M, Fiks I et al. Trans-illumination fluorescence imaging of deep-seated tumors in small animals. Photonics Lasers Med. 4(1), 85 (2015).

17 Harisinghani MG, Barentsz J, Hahn PF et al. Noninvasive detection of clinically occult lymph-node metastases in prostate cancer. N. Engl. J. Med. 348(25), 2491-2499 (2003).

18 Wang X, Niu D, Li P et al. Dual-enzyme-loaded multifunctional hybrid nanogel system for pathological responsive ultrasound imaging and T2-weighted magnetic resonance imaging. ACS Nano 9(6), 5646-5656 (2015).

19 Kellnberger S, Rosenthal A, Myklatun A, Westmeyer GG, Sergiadis G, Ntziachristos V. Magnetoacoustic sensing of magnetic nanoparticles. Phys. Rev. Lett. 116(10), 108103 (2016).

20 Kilcoyne A, Harisinghani MG, Mahmood U. Prostate cancer imaging and therapy: potential role of nanoparticles. J. Nucl. Med. 57(Suppl. 3), S105-S110 (2016). 